\title{
Integrated Estimation Structure for the Tire Friction Forces in Ground Vehicles
}

\author{
E. Hashemi, M. Pirani, A. Khajepour, B. Fidan, A. Kasaiezadeh, S-K. Chen, and B. Litkouhi
}

\begin{abstract}
This paper presents a novel corner-based force estimation method to monitor tire capacities required for the traction and stability control systems. This is entailed for more advanced vehicle stability systems in harsh maneuvers. A novel estimation structure is proposed in this paper for the longitudinal, lateral, and vertical tire forces robust to the road friction condition. A nonlinear and a Kalman observer is utilized for estimation of the longitudinal and lateral friction forces. The stability and performance of the time-varying estimators are explored and it is shown that the developed integrated structure is robust to model uncertainties and does not require knowledge of the road friction. The proposed method is experimentally tested in several maneuvers on different road surface conditions and the results illustrate the accuracy and robustness of the state estimators.
\end{abstract}

\section{INTRODUCTION}

Advanced vehicle stability control and active safety systems require dependable vehicle states, which may not be readily accessible by measurements, thus needing to be estimated. One major practical issue that has dominated the vehicle state estimation field is a robust tire friction force estimation. Several studies first have focused on road friction estimation and identification of tire parameters, in order to estimate longitudinal and lateral tire forces. Alvarez et al. [1] used a parameter adaptation law, a Lyapunovbased state estimator, and the dynamic LuGre model [2] to estimate the road friction and longitudinal forces during an emergency brake condition. Employing the equivalent output error injection approach, Patel et al. proposed a second-order and third-order sliding mode observers in [3] to estimate the friction coefficient and consequently tire forces during brake conditions on the pseudostatic LuGre [4], dynamic LuGre, and parameter-based friction [5] models. Ghandour et al. [6] developed a force and road friction estimation structure based on an iterative quadratic minimization of the error between the developed lateral force estimator and the tire/road interaction Dugoff model. Rajamani et al. [7] suggest a recursive least square for road identification and a nonlinear observer for longitudinal force estimation having wheel torques and accurate slip-ratio data from GPS. These methods rely on simultaneous road condition identification, which may impose undesirable estimation error produced by the time-varying model parameters.

A. Khajepour, B. Fidan, E. Hashemi, and M. Pirani are with the Department of Mechanical and Mechatronics Eng., University of Waterloo, Waterloo, ON, Canada (e-mail: a.khajepour, fidan, ehashemi, mpirani@uwaterloo.ca). B. Litkouhi, A. Kasaiezadeh, S-K. Chen, and are with the General Motors RD, Warren, MI, USA (e-mail: bakhtiar.litkouhi, alireza.kasaiezadeh, shih-ken.chen@gm.com).
Estimation of longitudinal and lateral forces independent from the road condition may be classified on the basis of wheel dynamics and planar kinetics into the nonlinear, sliding mode, Kalman-based, and unknown input observers. A force estimation method based on the steering torque measurement is introduced in [8], which requires additional measurements. Hsu et al. provide a nonlinear observer to estimate tire slip angles as well as the road friction condition in [9] with steering torque measurement. Baffet et al. [10] proposed a cascaded structure for estimation of the tire forces and vehicle side-slip angle with a sliding mode observer and extended Kalman Filter (EKF). Doumiati et al. [11] estimate tire forces with EKF and UKF. In their approach, longitudinal and lateral force evolution is modelled with a random walk model. They assume that longitudinal and lateral tire forces and force sums on each track are associated according to the dispersion of vertical forces. Cho et al. [12] estimate lateral tire forces using the planar kinetics and a randomwalk Kalman filter. A Kalman-based unknown input observer (UIO) is developed by Wang et al. [13], [14] for longitudinal and lateral force estimation with the wheel dynamics, planar kinetics, measured wheel speeds, wheel torques, and the yaw rate. Using UKF and the wheel dynamics, Hashemi et al. [15] developed a longitudinal force estimator robust to the road friction changes and uncertainties in the model such as i.e. effective rolling radius, measured wheel speed and torques. Similarly, employing UKF for an antilock braking control system, Sun et al. [16] propose a nonlinear observer robust to the road friction for the longitudinal force and slip ratio estimation during brake. Their approach is tested during brake maneuvers on different road conditions.

In the following, a corner-based methodology for estimation of the longitudinal forces based on a nonlinear observer is first discussed, then an adaptive Kalman-based lateral force estimation is proposed in Section III. Vertical force estimation is also provided in Section III. Section IV presents experimental and simulation results used to corroborate the approach on different road conditions and in various maneuvers with high and low longitudinal/lateral excitations. Finally, conclusions are provided in Section V.

\section{Problem Statement}

Tire forces exhibit the vehicles capacity to perform requested maneuvers and provide information about the stability of the vehicle. Since tire force calculation requires road friction information, even accurate slip ratio/angle information from high precision GPS and high fidelity tire model will not lead to tire forces at each wheel. Therefore, estimation 
of the longitudinal, lateral, and vertical tire forces using measurements available on current production vehicles, yet robust to different road conditions has been the main focus of related literature in recent years, and the topic of this study.

Longitudinal force estimation significantly contributes to model-based velocity estimators [17] and traction control systems. Neglecting the bearing's viscous damping, one can write the wheel dynamic equation as follows with $i \in\{f, r\}$ (front and rear axles) and $j \in\{L, R\}$ (left and right tires):

$$
F_{x_{i J}}=\frac{1}{R_{e}}\left(T_{t_{i J}}-I_{w} \dot{\omega}_{i J}\right)+\Omega_{x}
$$

where $R_{e}$ is the wheel effective rolling radius, $T_{t}$ represents the total effective torque on the wheel, $F_{x}$ is the longitudinal tire force, $\dot{\omega}$ is the wheel acceleration, $I_{w}$ is the wheel's moment of inertia and $\Omega_{x}$ represents uncertainties in the model including the effective radius, torque, etc.

Model-based stability control systems and lateral velocity estimators use lateral forces at each corner. The sum of longitudinal/lateral forces at each axle i.e. $F_{x_{i}}=\sum_{J} F_{x_{i J}}$ and $F_{y_{i}}=\sum_{J} F_{y_{i J}}$ are utilized for the longitudinal and lateral dynamics:

$$
\begin{aligned}
& m \check{a}_{x}=F_{x_{f}} \cos \delta-F_{y_{f}} \sin \delta+F_{x_{r}}+\Omega_{f_{x}} \\
& m \check{a}_{y}=F_{y_{f}} \cos \delta+F_{x_{f}} \sin \delta+F_{y_{r}}+\Omega_{f_{y}}
\end{aligned}
$$

in which $\delta$ is the steering angle (with parallel steering in front wheels) and $\Omega_{f_{x}}, \Omega_{f_{y}}$ represent longitudinal and lateral uncertainties due to the acceleration measurement, geometry, and forces. The measured longitudinal and lateral accelerations include the kinematics of the vehicle's CG $V_{x}, V_{y}$, vehicle's body pitch/roll angles $\theta_{v}, \phi_{v}$, and road grade/bank angles $\theta_{r}, \phi_{r}$. Therefore, acceleration measurements $a_{x}, a_{y}$ are corrected with the road and body's roll/pitch angles as $\check{a}_{x}=a_{x}-g \sin \theta_{t}$ and $\check{a}_{y}=a_{y}-g \sin \phi_{t}$ where $\theta_{t}=\theta_{v}+\theta_{r}$ and $\phi_{t}=\phi_{v}+\phi_{r}$.

The derivative of the yaw rate $r$ is also related to the sum of forces at each axles as:

$$
\begin{aligned}
I_{z} \dot{r} & =\left(F_{y_{f}} \cos \delta+F_{x_{f}} \sin \delta\right) d_{f} \\
& +\left(\bar{F}_{x_{f}} \cos \delta-\bar{F}_{y_{f}} \sin \delta\right) \frac{T_{r_{f}}}{2}-F_{y_{r}} d_{r}+\bar{F}_{x_{r}} \frac{T_{r_{r}}}{2}+\Omega_{r}
\end{aligned}
$$

in which $T_{r_{f}}, T_{r_{r}}$ are the length of front and rear tracks respectively, the distances from the front and rear axles' to CG are denoted by $d_{f}, d_{r}$, and $\bar{F}_{x_{i}}=F_{x_{i R}}-F_{x_{i L}}, \bar{F}_{y_{i}}=$ $F_{y_{i R}}-F_{y_{i L}}$. Uncertainties due to the CG location, yaw rate measurement, and forces are represented by $\Omega_{r}$. The effects of the vehicle body's vertical motion and the roll/pitch angles are not commonly considered in the existing vertical force estimation methods [11], [12]. To tackle this issue, the vertical force estimator module is developed in this paper using lateral and longitudinal vehicle dynamics and incorporation of the vehicle angles $\phi_{v}, \theta_{v}$ from [18]. Normal forces at each axle/tire and the longitudinal, lateral, and vertical components of the accelerations in the vehicle chassis coordinates, i.e., $\tilde{a}_{\theta x}, \tilde{a}_{\theta z}$ and $\tilde{a}_{\phi y}, \tilde{a}_{\phi z}$ are schematically illustrated in Fig. 1.

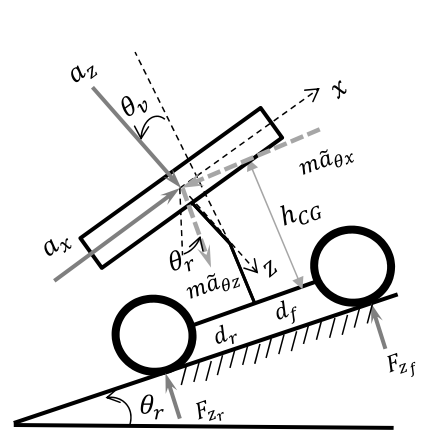

(a)

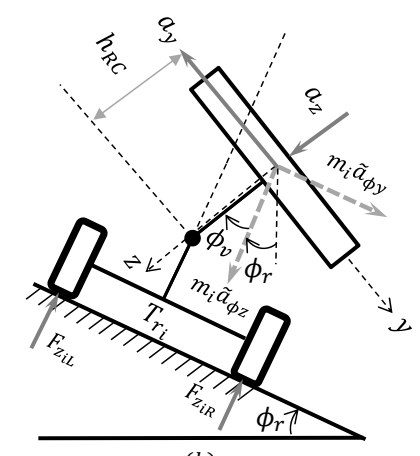

(b)
Fig. 1: (a) pitch model (b) roll model

In the following section an estimation structure robust to the road condition changes is introduced and experimentally tested for tire forces. This estimation structure can be utilized in any vehicle's active safety system or model-based velocity estimator.

\section{INTEGRATED ForCE Estimation MODEL}

The developed longitudinal and lateral force observers use accelerations, wheel speed, and wheel torques to estimate the tire forces at all four corners without any road friction data or any limiting assumption. Vertical forces are also estimated using vehicle body dynamics, the load transfer, and the measured accelerations.

\section{A. Longitudinal force estimation}

The corner-based estimation approach proposed in this section for the longitudinal force estimation, uses a PID state observer [19]-[21] that has also been used in other applications. The longitudinal force estimate is expressed as:

$$
\hat{F}_{x_{i J}}=\frac{T_{t_{i J}}-I_{w} \dot{\omega}_{i J}}{R_{e}}-k_{1} \tilde{\omega}_{i J}+k_{3} \int \tilde{F}_{x_{i J}} d t
$$

where $k_{1}, k_{3}$ are design parameters. The estimated wheel speed $\hat{\omega}$ at each corner $i J$ is described as:

$$
\dot{\hat{\omega}}_{i J}=\frac{1}{I_{w}}\left(T_{t_{i J}}-R_{e} \hat{F}_{x_{i J}}+k_{2} \int \tilde{\omega}_{i J} d t+R_{e} k_{3} \int \tilde{F}_{x_{i J}} d t\right)
$$

in which $k_{2}$ is a design parameter, $\tilde{\omega}_{i J}=\omega_{i J}-\hat{\omega}_{i J}$, and $\tilde{F}_{x_{i J}}=F_{x_{i J}}-\hat{F}_{x_{i J}}$ is the longitudinal force estimation error.

Theorem 1: The error dynamics for the longitudinal estimator (4) on the wheel dynamics with time-varying parameter $\omega_{i J}$ is exponentially stable.

Proof: : Subtracting the longitudinal force (1) from the estimated longitudinal force (4) leads to the estimation error $\tilde{F}_{x_{i J}}$. This force estimation error can be described as $\tilde{F}_{x_{i J}}=$ $-k_{3} \int \tilde{F}_{x_{i J}} d t+k_{1} \tilde{\omega}_{i J}+\Omega_{x}$. The time derivative of the error dynamic yields $\dot{\tilde{F}}_{x_{i J}}=A_{f} \tilde{F}_{x_{i J}}+B_{f} \dot{\tilde{\omega}}_{i J}+\dot{\Omega}_{x}$ with $A_{f}=$ 
$-k_{3}$ and $B_{f}=k_{1}$. The discretized form of the longitudinal force error dynamics is:

$$
\dot{\tilde{F}}_{x_{i J_{k+1}}}=A_{f_{d}} \tilde{F}_{x_{i J_{k}}}+B_{f_{d}} \dot{\tilde{\omega}}_{i J_{k}}+\dot{\Omega}_{x_{k}}
$$

where $A_{f_{d}}, B_{f_{d}}$ are the discretized state and input matrices.

Remark 1: In general, discretization of the continuoustime system $\dot{x}=A_{c} x+B_{c} u$ with the output $y=C_{c} x+$ $D_{c} u$ is done by the Step-Invariance method [22], because of its precision and response characteristics. Input to the continuous-time system is the hold signal $u_{k}=u\left(t_{k}\right)$ for a period between $t_{k} \leq t<t_{k+1}$ with the sample time $T_{s}$. Then, the discrete-time system $x_{k+1}=A_{d} x_{k}+$ $B_{d} u_{k}, y_{k}=C_{d} x_{k}+D_{d} u_{k}$ has the output matrices $C_{d}=$ $C_{c}, D_{d}=D_{c}$ and state/input matrices $A_{d}=e^{A_{c}(t) T_{s}}, B_{d}=$ $\int_{0}^{T_{s}} e^{A_{c}(t) \tau} B_{c}(t) d \tau$.

Substituting $\hat{F}_{x_{i J}}$ from (4) in (5), one can rewrite:

$$
\dot{\hat{\omega}}_{i J}=\dot{\omega}_{i J}+\frac{1}{I_{w}}\left(R_{e} k_{1} \tilde{\omega}_{i J}+k_{2} \int \tilde{\omega}_{i J} d t\right) .
$$

The deviation of the estimated wheel speed from the measured one is denoted by $\tilde{\omega}$ and incorporated for the force estimation as in (4). Subtracting the estimated wheel speed (5) from the wheel speed by the wheel dynamics (1) results in $I_{w} \dot{\tilde{\omega}}_{i J}=-R_{e} \tilde{F}_{x_{i J}}+R_{e} \Omega_{x}-k_{2} \int \tilde{\omega}_{i J}-R_{e} k_{3} \int \tilde{F}_{x_{i J}} d t$. Taking time derivative and replacing the error dynamics $\dot{\tilde{F}}_{x_{i J}}=-k_{3} \tilde{F}_{x_{i J}}+k_{1} \dot{\tilde{\omega}}_{i J}+\dot{\Omega}_{x}$ leads to $I_{w} \ddot{\tilde{\omega}}_{i J}+R_{e} k_{1} \dot{\tilde{\omega}}_{i J}+$ $k_{2} \tilde{\omega}_{i J}=0$ which is in the discretized state space form:

$$
\mathbf{x}_{w_{k+1}}=A_{w_{d}} \mathbf{x}_{w_{k}}
$$

with states $\mathbf{x}_{w}=\left[\begin{array}{cc}\tilde{\omega} & \dot{\tilde{\omega}}\end{array}\right]^{T}$. The discretized state matrix is $A_{w_{d}}=e^{A_{w} T_{s}}$ where:

$$
A_{w}=\left[\begin{array}{cc}
0 & 1 \\
\frac{-k_{2}}{I_{w}} & \frac{-R_{e} k_{1}}{I_{w}}
\end{array}\right] .
$$

The matrix $A_{w}$ is Hurwitz and (9) is exponentially stable given $k_{1}, k_{2}>0$, therefore, $\mathbf{x}_{w} \rightarrow 0$ (i.e. $\tilde{\omega}_{i J} \rightarrow 0$ and $\dot{\tilde{\omega}}_{i J} \rightarrow 0$ ). This means that the estimation error dynamics (6) changes to $\tilde{F}_{x_{i J_{k+1}}}=A_{f_{d}} \tilde{F}_{x_{i J_{k}}}+\dot{\Omega}_{x_{k}}$ that is an exponentially stable dynamic for $\forall k_{3}>0$ and has attenuation for uncertainties with the gain $\frac{1}{k_{3}}$.

\section{B. Lateral force estimation}

Longitudinal forces at each corner i.e. $F_{x_{i J}}$ are assumed to be available from (4) in the previous subsection. Set of equations (3) together with the longitudinal and lateral dynamics can be solved for the lateral forces $F_{y_{i J}}$ at each corner with the assumption of lateral force distribution based on the normal forces, but this may not address maneuvers in which road friction under each tire is different. To resolve this, a method for the lateral force estimation is proposed in this section that uses longitudinal forces and accelerations $a_{x}, a_{y}$ and the yaw rate $r$ measurements from a 3 -axis IMU.

The set of equations (3) can be rewritten in the following lateral force estimator with states $\mathbf{x}=\left[\begin{array}{lll}F_{y_{f}} & F_{y_{r}} & \bar{F}_{y_{f}}\end{array}\right]^{T}$ and output (measurement) $\mathbf{y}=\left[\begin{array}{lll}\check{a}_{x} & \check{a}_{y} & r\end{array}\right]^{T}$ :

$$
\begin{aligned}
& \dot{\mathbf{x}}=A_{y} \mathbf{x}+\boldsymbol{\Omega}_{y}, \\
& \mathbf{y}=C(\delta) \mathbf{x}+\mathbf{u}_{y}+\boldsymbol{\Gamma}_{y}
\end{aligned}
$$

where $A_{y}=\mathbf{0}_{3 \times 3}$ and uncertainties in the process and measurements are denoted by $\boldsymbol{\Omega}_{y}$ and $\boldsymbol{\Gamma}_{y}$. The output matrix $C(\delta)$ and $\mathbf{u}_{y}$ are defined as:

$$
\left.\begin{array}{c}
C(\delta)=\left[\begin{array}{ccc}
-\frac{1}{m} \sin \delta & 0 & 0 \\
\frac{1}{m} \cos \delta & \frac{1}{m} & 0 \\
\frac{1}{I_{z}} \cos \delta d_{f} & -\frac{1}{I_{z}} d_{r} & \frac{1}{2 I_{z}} \sin \delta T_{r_{f}}
\end{array}\right], \\
\frac{1}{m}\left(F_{x f} \cos \delta+F_{x r}\right) \\
\frac{1}{m} F_{x f} \sin \delta \\
\frac{1}{I_{z}}\left(F_{x f} \sin \delta d_{f}+\bar{F}_{x f} \cos \delta \frac{T_{r_{f}}}{2}+\bar{F}_{x r} \frac{T_{r_{r}}}{2}\right)
\end{array}\right],
$$

The matrix $C(\delta)$ is time-varying and physically bounded (because of the steering angle and its derivative).

Stability of the estimator: Observability is a sufficient condition for implementation of an optimal variance filter (such as a Kalman estimator). Therefore, a Kalman-based observer can be employed on system (10) with the discretized form of:

$$
\begin{aligned}
\mathbf{x}_{k+1} & =A_{y k} \mathbf{x}_{k}+\boldsymbol{\Omega}_{y k} \\
\mathbf{y} & =C_{k} \mathbf{x}+\mathbf{u}_{y k}+\boldsymbol{\Gamma}_{y k},
\end{aligned}
$$

which have the noise covariances $Q_{y k}=$ $\mathbb{E}\left[\boldsymbol{\Omega}_{y k}, \boldsymbol{\Omega}_{y k}^{T}\right], R_{y k}=\mathbb{E}\left[\boldsymbol{\Gamma}_{y k}, \boldsymbol{\Gamma}_{y k}^{T}\right]$ for the model and measurements, respectively. Process and measurement noises are assumed to be uncorrelated $\mathbb{E}\left[\boldsymbol{\Omega}_{y k}, \boldsymbol{\Gamma}_{y k}{ }^{T}\right]=0$ and have zero mean $\mathbb{E}\left[\boldsymbol{\Omega}_{y k}\right]=\mathbb{E}\left[\boldsymbol{\Gamma}_{y k}\right]=0 ; \forall k \in \mathbb{N}$.

The discrete-time Kalman observer suggests the following prediction with correction to estimate the states defined by $\hat{\mathbf{x}}_{k+1 \mid j} \triangleq \mathbb{E}\left[\mathbf{x}_{k} \mid y_{j}\right]$ using a sequence of measurements $y_{j}$ :

$$
\hat{\mathbf{x}}_{k+1 \mid k}=A_{y k} \hat{\mathbf{x}}_{k \mid k-1}+K_{k}\left(\mathbf{y}_{k}-C_{k} \hat{\mathbf{x}}_{k \mid k-1}\right),
$$

where the optimal Kalman gain is $K_{k}=$ $A_{y k} P_{k \mid k-1} C_{k}^{T}\left(C_{k} P_{k \mid k-1} C_{k}^{T}+R_{y k}\right)^{-1}$ and error covariance $P_{k+1 \mid k} \triangleq \operatorname{cov}\left(\mathbf{x}_{k+1}-\hat{\mathbf{x}}_{k+1 \mid k}\right)$ forms a discrete time-varying Riccati equation (14) for both zero and non-zero state initialization $\hat{\mathbf{x}}_{0 \mid-1}=\mathbb{E}\left[\mathbf{x}_{0}\right]$ :

$$
P_{k+1 \mid k}=A_{y k} P_{k \mid k-1} A_{y k}^{T}+Q_{y k}-K_{k} C_{k} P_{k \mid k-1} A_{y k}^{T},
$$

where the state covariance is initialized as $P_{0 \mid-1} \triangleq$ $\operatorname{cov}\left(\mathbf{x}_{0}\right)=\mathbb{E}\left[\left(\mathbf{x}_{0}-\hat{\mathbf{x}}_{0 \mid-1}\right)\left(\mathbf{x}_{0}-\hat{\mathbf{x}}_{0 \mid-1}\right)^{T}\right]$. The estimation error is defined by $\mathbf{e}_{k+1 \mid j} \triangleq \mathbf{x}_{k+1}-\hat{\mathbf{x}}_{k+1 \mid j}$, which yields:

$$
\mathbf{e}_{k+1 \mid k}=\left(A_{y k}-K_{k} C_{k}\right) \mathbf{e}_{k \mid k-1}-K_{k} \boldsymbol{\Omega}_{y k}+\boldsymbol{\Gamma}_{y k} .
$$

The observability matrix for the system (10) can be written as [23]:

$$
\begin{array}{r}
\mathcal{O}_{n}=\left[\begin{array}{lll}
\tau_{1} & \tau_{2} \ldots & \tau_{n}
\end{array}\right]^{T} \\
\tau_{1}=C, \quad \tau_{i+1}=\tau_{i} A_{y}(t)+\dot{\tau}_{i},
\end{array}
$$

Observability of the system (10) is confirmed by holding the full rank condition $\operatorname{rank}\left(\mathcal{O}_{3}\right)=3$ for the operating regions of the steering angle and its time derivatives. Therefore, the 
presented model-based estimation is stable, and errors of the state mean have bounded variance for zero and non-zero initial covariances.

\section{Vertical force estimation}

Estimation of the vertical forces at each corner is addressed in this subsection using lateral and longitudinal vehicle dynamics and the sprung mass angles. The sprung mass roll and pitch angles $\phi_{v}, \theta_{v}$ are not achievable by integration over the roll and pitch rate signals $\dot{\phi}_{m}, \dot{\theta}_{m}$ because of sensor drift. Rehm provided a linear observer with low-pass filtering in [18] to estimate the vehicle body's roll/pitch angles as $\dot{\hat{\phi}}_{v}=\dot{\phi}_{m}+L_{\phi} e_{\phi}, \dot{\hat{\theta}}_{v}=\dot{\theta}_{m}+L_{\theta} e_{\theta}$ by compensation over the error between the filtered estimates and the stationary roll/pitch values i.e. $e_{\phi}, e_{\theta}$ and the observer gains $L_{\phi}, L_{\theta}$.

The longitudinal and vertical acceleration components of the longitudinal dynamics are defined as $\tilde{a}_{\theta x}=a_{x} \cos \theta_{v}+$ $a_{z} \sin \theta_{v}$ and $\tilde{a}_{\theta z}=a_{z} \cos \theta_{v}-a_{x} \sin \theta_{v}$ where $a_{x}, a_{z}$ are the measured longitudinal and vertical accelerations by an IMU attached to the sprung mass, which are affected by the kinematics of the vehicle's CG, vehicle pitch angle $\theta_{v}$, and the road grade angle $\theta_{r}$. Normal forces at front and rear axles, thus can be calculated by:

$$
\begin{aligned}
& F_{z_{f}}=-\frac{m}{\left(d_{f}+d_{r}\right)}\left(h_{C G} \tilde{a}_{\theta x}-d_{r} \tilde{a}_{\theta z}\right) \\
& F_{z_{r}}=\frac{m}{\left(d_{f}+d_{r}\right)}\left(h_{C G} \tilde{a}_{\theta x}+d_{f} \tilde{a}_{\theta z}\right),
\end{aligned}
$$

where the height of the vehicle's center of gravity is $h_{C G}$, and $d_{w b}=d_{f}+d_{r}$ is the wheel base. Similarly, the lateral and vertical acceleration components of the lateral dynamics are defined by $\tilde{a}_{\phi y}=a_{y} \cos \phi_{v}+a_{z} \sin \phi_{v}$ and $\tilde{a}_{\phi z}=$ $a_{z} \cos \phi_{v}-a_{y} \sin \phi_{v}$ in which $a_{y}$ is the measured lateral acceleration by IMU, which contains the kinematics of the vehicle's CG, vehicle roll $\phi_{v}$ angle, and the road bank angle $\phi_{r}$. Therefore, using (17) and defining equivalent masses at each axle $m_{i}=\frac{F_{z_{i}}}{g}, i \in\{f, r\}$ (front and rear axles), normal forces at each corner become:

$$
\begin{aligned}
& F_{z_{i L}}=\frac{m_{i}}{T_{r_{i}}}\left[\tilde{a}_{\phi z}\left(\frac{T_{r_{i}}}{2}-h_{R C} \sin \phi_{v}\right)-\tilde{a}_{\phi y} h_{C G}\right] \\
& F_{z_{i R}}=\frac{m_{i}}{T_{r_{i}}}\left[\tilde{a}_{\phi z}\left(\frac{T_{r_{i}}}{2}+h_{R C} \sin \phi_{v}\right)+\tilde{a}_{\phi y} h_{C G}\right],
\end{aligned}
$$

in which, the left and the right sides are denoted by $L, R$ and $h_{r c}$ is the height of the roll center. The proposed approach is experimentally validated on various road conditions using a full size test vehicle.

\section{EXPERIMENTS AND SIMULATIONS}

Experimental and simulation results are presented in this section to validate the proposed approach for the tire force estimation robust to the road friction changes. Road tests are conducted on an instrumented vehicle with four independent wheel drive. The vehicle specification are: mass $m=2275[\mathrm{~kg}]$, moment of inertia around yaw axis $I_{z}=$ $4650\left[\mathrm{~kg} \cdot \mathrm{m}^{2}\right]$, wheel moment of inertia $I_{w}=1.7\left[\mathrm{~kg} \cdot \mathrm{m}^{2}\right]$, front/rear axles to $\mathrm{CG} d_{f}=1.42, d_{r}=1.43[\mathrm{~m}]$, front/rear track widths $T_{r_{f}}=1.62, T_{r_{r}}=1.56[\mathrm{~m}], \mathrm{CG}$ and roll axis heights $h_{C G}=0.647, h_{R C}=0.54[\mathrm{~m}]$, and tires effective rolling radius $R_{e}=0.346[\mathrm{~m}]$.

The estimation modules require longitudinal and lateral accelerations, yaw rate, wheel speed as well as the wheel torques, which are measured using an IMU, regular ABS wheel speed sensors, and electric actuators respectively. Measured signals are communicated using a CAN-bus. The sampling time for the experiment is set to be $T_{s}=0.005[\mathrm{~s}]$. Real-time acquisition and processing of sensory information

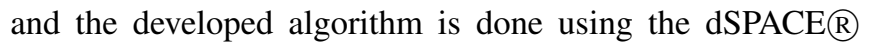
MicroAutobox.

Identified as hard driving conditions for evaluating state estimators or controllers, acceleration-in-turn (AiT) has been conducted on dry and slippery road conditions in the CarSim. The developed force estimators are tested in the CarSim model and the outcomes (Estimation) are compared in Fig. 2 with the measured tire forces by the CarSim (Measurement, CarSim) on dry and slippery roads. Accelerator is applied to $100 \%$ at $t=2[s]$ and continued to $t=4[s]$. It is released between 4 and $6[s]$ and pushed up to $100 \%$ again as a step signal till $t=11[s]$, then it is linearly reduced to zero at $t=15[\mathrm{~s}]$. A steering angle $\delta_{s w}=1[\mathrm{rad}]$ is imposed between $t=2-12[s]$ as well and the steering ratio is $r_{\delta}=16.5$.

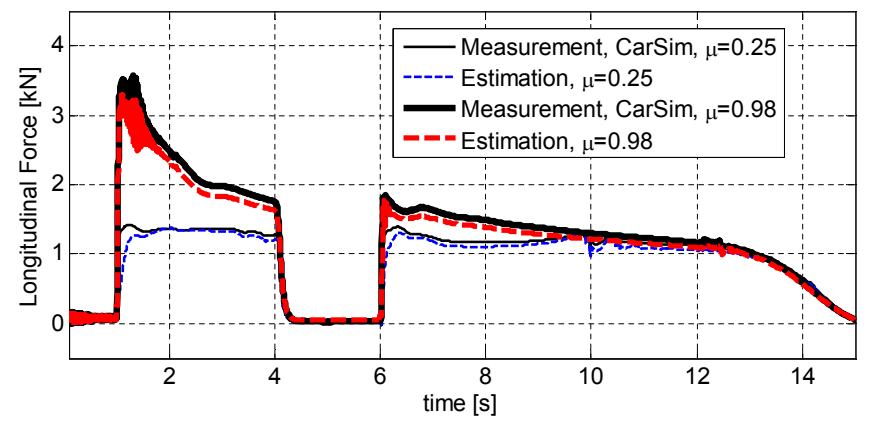

Fig. 2: Estimation results, AiT on dry and slippery roads

The fluctuations observed in the force profile curves existing in the CarSim results are attributed to the requested acceleration with high magnitude.

A harsh lane change (LC) maneuver is performed on a wet surface with $\mu \approx 0.45$ to evaluate the proposed force estimation approach in combined-slip conditions on the test platform vehicle. The experimental results of the rear left wheel are demonstrated in Fig. 3. The Effective torque $T_{t_{r j}}$ and the wheel speed $\omega_{r j}$ of the rear wheels are also depicted in Fig. 3.

The selected gains for these road experiments are $k_{1}=$ $3.41, k_{2}=16.22$ and $k_{3}=67.84$. The suggested longitudinal observer (4) also exhibits consistent results for other road experiments such as double-lane-change (DLC) and AiT on various road frictions as can be seen in Table I.

Performance of the lateral and vertical force estimator on dry and slippery surfaces is examined in several road experiments with the process and measurement noise covariance matrices $Q_{y}=0.11^{2} I_{3 \times 3}, R_{y}=0.018^{2} I_{3 \times 3}$ which 

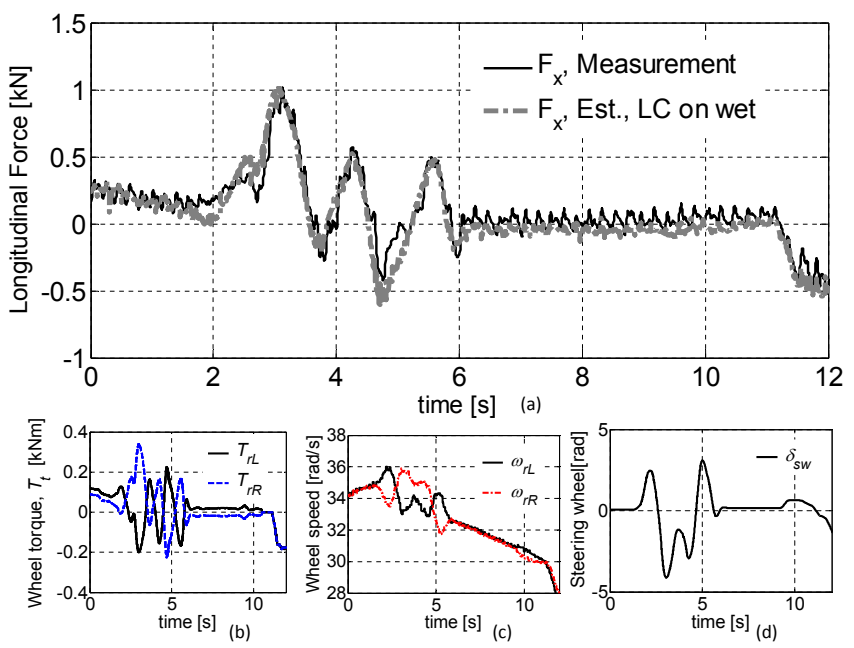

Fig. 3: AWD, lane change on wet (a) estimated $\hat{F}_{x}$ at rL (b) wheel torques (c) wheel speeds (d) steering wheel angle

change appropriately upon detection of a high-slip case. The high-slip detection algorithm utilizes the wheel angular acceleration at each corner to remove the outliers and detect the large slip scenarios. The rates of the wheel speed are then checked locally with a threshold $\dot{\omega}_{t h}$ and the covariance matrices change at each corner for the lateral force estimation when the criteria $\left|\dot{\omega}_{i j}\right| \geq\left|\dot{\omega}_{t h}\right|$ is met. Simulation and experimental results confirm the validity of the approach on dry and slippery roads.

Results of the proposed force estimator in lane change on the dry road are presented in Fig. 4 and compared with the measurement for the all-wheel drive (AWD) traction configuration. The measured accelerations and yaw rate $r$ are also provided to show the characteristics of the maneuver.
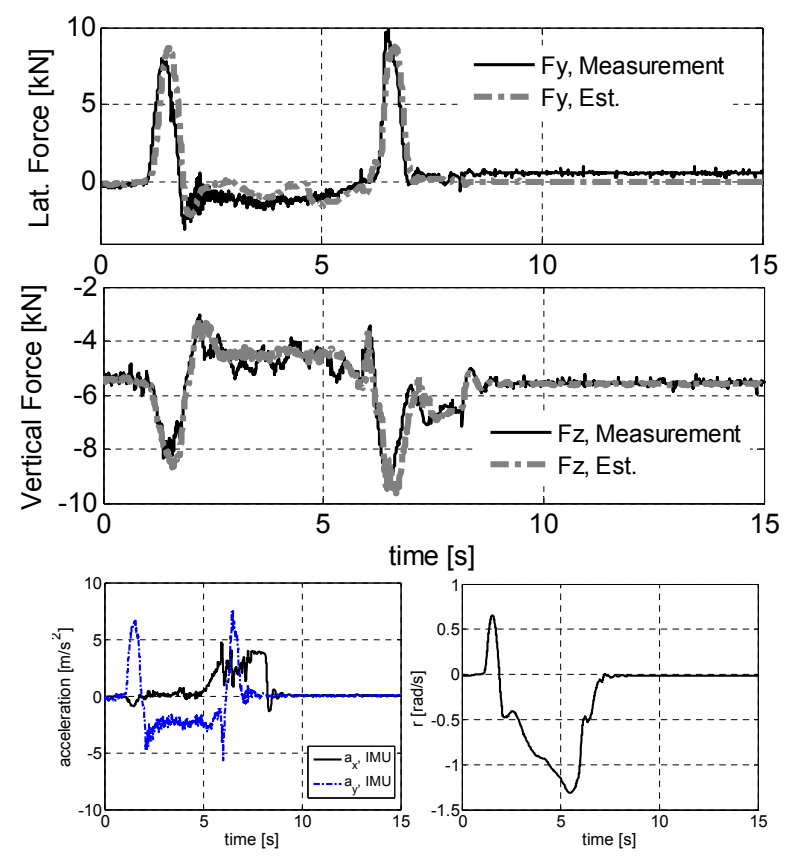

Fig. 4: Lateral and vertical force estimates, LC on a dry
A harsh steering on an icy road is done and the results of the front left corner are illustrated for the AWD case in Fig. 5. The maneuver ended up on a surface with packed snow which is highly slippery itself with $\mu \approx 0.3$. The fluctuations observed in the measured force profile are attributed to the low-stick characteristics of the packed snow.
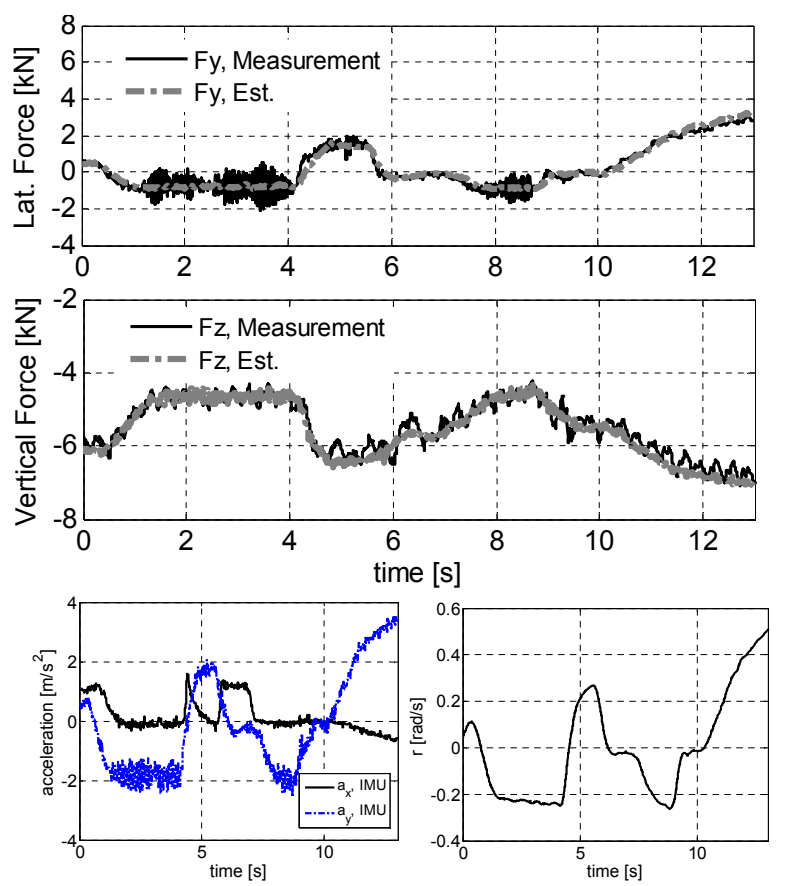

Fig. 5: Force estimates, steering on ice then packed snow

The process and measurement covariance matrices changes to $Q_{y}=0.036^{2} I_{3 \times 3}, R_{y}=0.4^{2} I_{3 \times 3}$ for very high longitudinal slip conditions (based on $\dot{\omega}_{i j}$ ).

Accuracy of the force and velocity estimators are evaluated in different maneuvers with the normalized root mean square (NRMS) of the error defined by $\rho_{e}=\frac{\sqrt{\sum_{i=1}^{N_{s}}\left(\hat{\tau}_{i}-\tau_{i}\right)^{2} / N_{\tau}}}{\bar{\tau}}$, where the estimated and measured signals are denoted by $\hat{\tau}$ and $\tau$, respectively, $N_{\tau}$ is the number of collected signal samples during a driving scenario (DLC, BiT, LC etc.), and $\bar{\tau}=\max _{i=1 \ldots N_{\tau}}\left|\tau_{i}\right|$ shows the maximum value of the measured signal. Table I provides the NRMS and maximum values of the vertical, longitudinal, and lateral forces in different driving scenarios and on various road frictions.

As can be seen from Table I, the NRMS of the estimated vertical, longitudinal, and lateral forces are less than $4.8 \%$, $6.4 \%$, and $7.2 \%$, respectively, for the performed maneuvers on dry and snowy roads. These normalized RMS of the errors corroborate the effectiveness of the algorithm for cornerbased force estimation on dry and slippery roads.

\section{CONCLUSION}

The force estimators exhibit accurate performance for the maneuver with the pure-slip characteristics (i.e. launch, normal driving, acceleration/deceleration) as well as the combined-slip ones (i.e. acceleration-in-turn and brake-inturn) on dry and slippery roads. 
TABLE I: NRMS of the errors for the force estimators

\begin{tabular}{l|cccc}
\hline \multirow{2}{*}{$\begin{array}{l}\text { Estimated } \\
\text { forces }\end{array}$} & \multicolumn{2}{|c}{ BiT/Accel., snow } & \multicolumn{2}{c}{ DLC on dry } \\
\cline { 2 - 5 }$\rho_{e}[\%]$ & $\bar{\tau}[N]$ & $\rho_{e}[\%]$ & $\bar{\tau}[N]$ \\
\hline$F_{z_{f L}}$ & 4.69 & 8634 & 2.82 & $1.28 e+4$ \\
$F_{z_{f R}}$ & 3.17 & 6415 & 4.75 & $1.23 e+4$ \\
$F_{z_{r L}}$ & 3.66 & 7093 & 3.31 & $1.03 e+4$ \\
$F_{z_{r R}}$ & 2.52 & 6261 & 2.18 & $1.07 e+4$ \\
\hline$F_{x_{f L}}$ & 5.11 & 2048 & 4.45 & 4746 \\
$F_{x_{f R}}$ & 5.04 & 2044 & 4.12 & 4039 \\
$F_{x_{r L}}$ & 6.17 & 731 & 4.68 & 3982 \\
$F_{x_{r R}}$ & 6.38 & 728 & 4.22 & 3979 \\
\hline$F_{y_{f L}}$ & 4.97 & 3171 & 3.10 & $1.01 e+4$ \\
$F_{y_{f R}}$ & 6.75 & 1187 & 3.04 & 9977 \\
$F_{y_{r L}}$ & 6.53 & 2746 & 4.08 & 9194 \\
$F_{y_{r R}}$ & 7.16 & 1425 & 2.92 & 8528 \\
\hline
\end{tabular}

The important feature of the proposed force estimator is that it does not implement any tire model and is independent from changes in the road friction or tire parameters due to wear, inflation pressure, temperature, etc. In addition, the suggested force estimators can address the cases in which tires are on surfaces with various road frictions since the adaptive Kalman-based lateral force estimator in (10) is developed without any assumption on the lateral force distribution. The proposed Kalman filter with adaptive covariance matrices based on the wheel rotational acceleration can handle dry and slippery roads with $\rho_{e}<7.2 \%$ for the lateral forces. Moreover, the longitudinal force estimator deal with the model uncertainties using the gains $k_{1}, k_{2}, k_{3}$ for an appropriate pole placement and robust observer design which leads to $\rho_{e}<6.4 \%$.

Observed errors between the measured and estimated forces may have several sources such as camber angle, inaccurate inertial parameters, and uncertainties in the CG location, which will be addressed in future.

\section{ACKNOWLEDGMENT}

The authors would like to acknowledge the financial support of the Automotive Partnership Canada, Ontario Research Fund, and the financial and technical support of General Motors Co.

\section{REFERENCES}

[1] L. Alvarez, J. Yi, R. Horowitz, and L. Olmos, "Dynamic friction model-based tire-road friction estimation and emergency braking control," Journal of dynamic systems, measurement, and control, vol. 127, no. 1, pp. 22-32, 2005.

[2] C. Canudas-de Wit, P. Tsiotras, E. Velenis, M. B. Gissinger, and G. Gissinger, "Dynamic Friction Models for Road/Tire Longitudinal Interaction," Vehicle System Dynamics, vol. 39, pp. 189-226, 2003.

[3] N. Patel, C. Edwards, and S. Spurgeon, "Tyreroad friction estimationa comparative study," Proceedings of the Institution of Mechanical Engineers, Part D: Journal of Automobile Engineering, vol. 222, no. 12 , pp. 2337-2351, 2008.

[4] C. Canudas-De-Wit, M. Petersen, and A. Shiriaev, "A New Nonlinear Observer for Tire/Road Distributed Contact Friction," in 42nd IEEE International Conference on Decision and Control (IEEE Cat. No.03CH37475), vol. 3, 2003, pp. 2246-2251.

[5] J. Yi, L. Alvarez, and R. Horowitz, "Adaptive emergency braking control with underestimation of friction coefficient," Control Systems Technology, IEEE Transactions on, vol. 10, no. 3, pp. 381-392, 2002.
[6] R. Ghandour, A. Victorino, M. Doumiati, and A. Charara, "Tire/road friction coefficient estimation applied to road safety," in Control \& Automation (MED), 2010 18th Mediterranean Conference on. IEEE, 2010, pp. $1485-1490$.

[7] R. Rajamani, G. Phanomchoeng, D. Piyabongkarn, and J. Y. Lew, "Algorithms for real-time estimation of individual wheel tire-road friction coefficients," Mechatronics, IEEE/ASME Transactions on vol. 17, no. 6, pp. 1183-1195, 2012.

[8] Y.-H. J. Hsu, "Estimation and control of lateral tire forces using steering torque," Ph.D. dissertation, Stanford University, 2009.

[9] Y.-H. J. Hsu, S. M. Laws, and J. C. Gerdes, "Estimation of tire slip angle and friction limits using steering torque," IEEE Transactions on Control Systems Technology, vol. 18, no. 4, pp. 896-907, 2010.

[10] G. Baffet, A. Charara, and D. Lechner, "Estimation of vehicle sideslip, tire force and wheel cornering stiffness," Control Engineering Practice, vol. 17, no. 11, pp. 1255-1264, 2009.

[11] M. Doumiati, A. Victorino, D. Lechner, G. Baffet, and A. Charara, "Observers for vehicle tyre/road forces estimation: experimental validation," Vehicle System Dynamics, vol. 48, no. 11, pp. 1345-1378, 2010.

[12] W. Cho, J. Yoon, S. Yim, B. Koo, and K. Yi, "Estimation of tire forces for application to vehicle stability control," Vehicular Technology, IEEE Transactions on, vol. 59, no. 2, pp. 638-649, 2010.

[13] Y. Wang, D. M. Bevly, and S.-k. Chen, "Longitudinal tire force estimation with unknown input observer," in ASME 2012 5th Annual Dynamic Systems and Control Conference joint with the JSME 2012 11th Motion and Vibration Conference. American Society of Mechanical Engineers, 2012, pp. 523-530.

[14] _ _Lateral tire force estimation with unknown input observer," in ASME 2012 5th Annual Dynamic Systems and Control Conference joint with the JSME 2012 11th Motion and Vibration Conference. American Society of Mechanical Engineers, 2012, pp. 531-538.

[15] E. Hashemi, A. Kasaiezadeh, A. Khajepour, N. Moshchuk, and S.-K Chen, "Robust estimation and experimental evaluation of longitudinal friction forces in ground vehicles," in ASME 2014 International Mechanical Engineering Congress and Exposition. American Society of Mechanical Engineers, 2014, pp. V012T15A021-V012T15A021.

[16] F. Sun, K. Lolenko, and J. Rudolph, "Nonlinear observer design for state estimation during antilock braking," Proceedings of the Institution of Mechanical Engineers, Part I: Journal of Systems and Control Engineering, vol. 228, no. 2, pp. 78-86, 2014.

[17] E. Hashemi, A. Kasaiezadeh, S. Khosravani, A. Khajepour, N. Moshchuk, and S.-K. Chen, "Estimation of Longitudinal Speed Robust to Road Conditions for Ground Vehicles," Vehicle System Dynamics, 2016.

[18] A. Rehm, "Estimation of vehicle roll angle," in Communications, Control and Signal Processing (ISCCSP), 2010 4th International Symposium on. IEEE, 2010, pp. 1-4.

[19] Y. Choi, "Pid state observer for robotic systems," in American Control Conference, 2006. IEEE, 2006, pp. 6-pp.

[20] A.-G. Wu, G.-R. Duan, and Y.-M. Fu, "Generalized pid observer design for descriptor linear systems," Systems, Man, and Cybernetics, Part B: Cybernetics, IEEE Transactions on, vol. 37, no. 5, pp. 13901395, 2007

[21] J. Y. Peng and X. B. Chen, "Integrated pid-based sliding mode state estimation and control for piezoelectric actuators," Mechatronics, IEEE/ASME Transactions on, vol. 19, no. 1, pp. 88-99, 2014.

[22] G. F. Franklin, J. D. Powell, and M. L. Workman, Digital Control of Dynamic Systems. Menlo Park: Addison Wesley Longman, 1998.

[23] R. Tóth, Modeling and identification of linear parameter-varying systems. Springer, 2010, vol. 403. 